http://jmscr.igmpublication.org/home/

ISSN (e)-2347-176x ISSN (p) 2455-0450

crossref DOI: https://dx.doi.org/10.18535/jmscr/v8i5.65

\author{
Journal Of Medical Science And Clinical Research \\ IGM Publication \\ An official Publication of IGM Publication
}

\title{
Nasopharyngeal Foreign Body in an Infant- A Cause for Inspiratory Stridor
}

Authors

\author{
Madhuri Arora ${ }^{1}$, Jagat Singh ${ }^{2}$, Lubhavni Dewan ${ }^{3}$, Obaidur Rehman $^{4}$, Rachna $^{5}$ \\ ${ }^{1,5}$ Post- graduate, Department of ENT, PT. B.D. Sharma PGIMS Rohtak, Haryana, India \\ ${ }^{2}$ Professor, Department of ENT, PT. B.D. Sharma PGIMS Rohtak, Haryana, India \\ ${ }^{3}$ Post- graduate, Department of Ophthalmology, Dr.Rajendra Prasad Centre For Ophthalmic Sciences, Delhi, India \\ ${ }^{4}$ Post- graduate, Department of Ophthalmology, Government Medical College and Hospital, Chandigarh, India
}

\begin{abstract}
Foreign bodies in the upper aero-digestive tract are a common paediatric emergency encountered, of them nasopharyngeal involvement is rarely seen. Here we present a case of an 8 month old infant presenting with stridor and a metallic round foreign body $(F B)$ in the nasopharynx and its rational management.

Keywords: nasopharyngeal foreign body, inspiratory stridor, infant, metallic round body.
\end{abstract}

\section{Introduction}

Foreign bodies in the upper aero-digestive tract are a very common pediatric emergency which are encountered routinely by the otorhinolaryngology department. They can either pass down the tract or can get stuck in the narrow regions such as nasal cavities, crico-esophageal junction. ${ }^{[1]}$ However, nasopharynx as a site for lodgment of foreign body is very rare and is seldom discussed even in standard otorhinolaryngology textbooks. ${ }^{[2]}$ Here, we present a case of an 8 month old infant who presented with inspiratory stridor to our emergency and was found to have a nasopharyngeal foreign body and discuss its management and possible outcomes.

\section{Case Report}

An 8 month old infant presented to the pediatric emergency with a history of acute onset inspiratory stridor. He had symptoms of upper respiratory tract infection for 3-4 days. An ENT opinion for upper airway assessment was taken. There was no history of any foreign body aspiration preceding the development of stridor. On eliciting further history the mother reported that the child has been breathing through mouth for the past 1 month. (Figure 1)

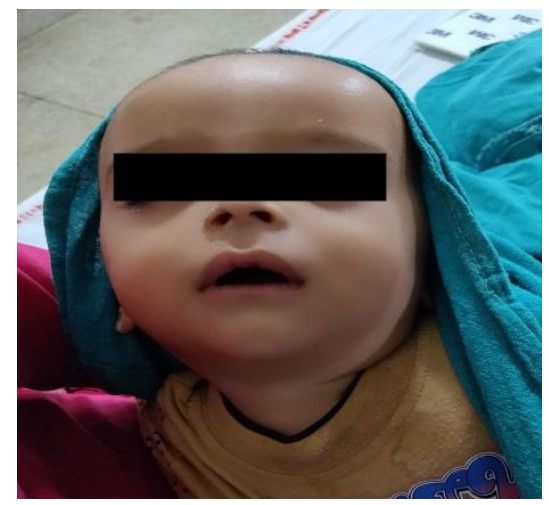

Fig. 1- An 8month old infant presented with mouth breathing and inspiratory stridor.

There was also one episode of a severe bout of cough 1 month back but no medical intervention was taken for the same. On examination, the child was afebrile, bilateral air entry was present and equal. On anterior rhinoscopy, the nasal mucosa 
was congested and nasal discharge was present. An $\mathrm{X}$-ray chest, nasopharynx and neck (soft tissues) both antero-posterior (AP) and lateral view were ordered for the patient which showed a round radioopaque shadow in the nasaopharynx. (Figure $2 \& 3$ )

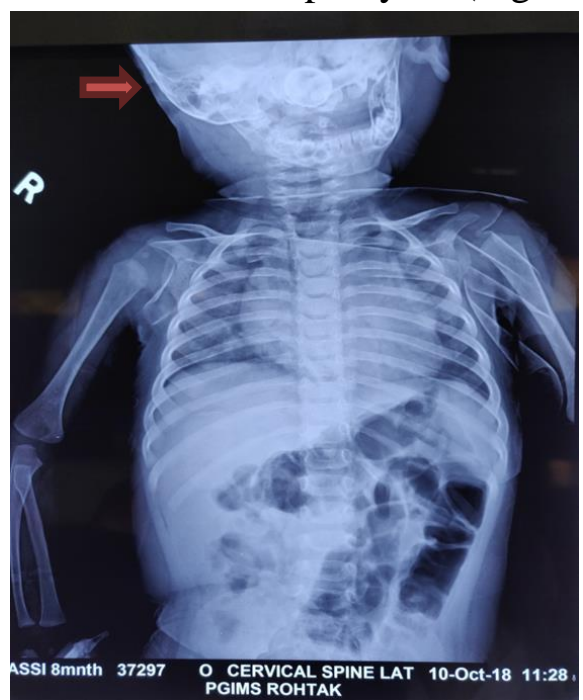

Fig.2- Showing AP view of Soft tissue neck along with nasopharynx and chest with radio opaque shadow seen above the level of palate

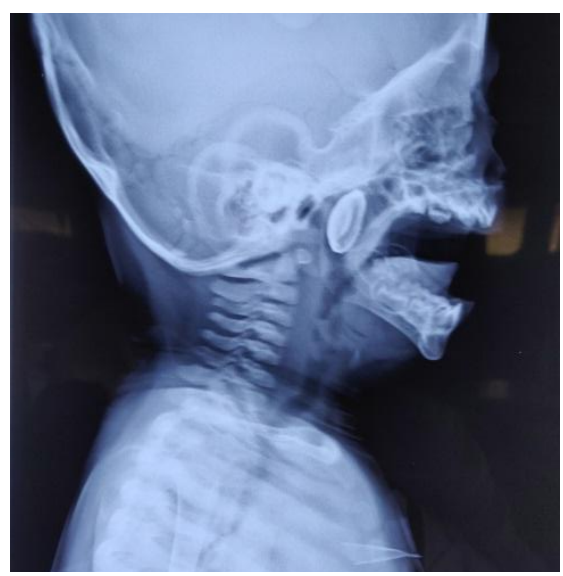

Fig.3- Lateral view X-ray of soft tissues neck along with nasopharynx with metallic foreign body seen in the nasopharynx just above the soft palate.

The child was planned for foreign body removal under general anaesthesia. Oro-tracheal intubation was performed after induction of general anaesthesia. Boyles-davis mouth gag was applied, after retracting the soft palate using foley's catheter. First pass using paediatric 0 degree endoscope was done to visualize the foreign body embedded in the nasopharynx. The foreign body was removed from the oral cavity using blakesley forceps. (Figure4)

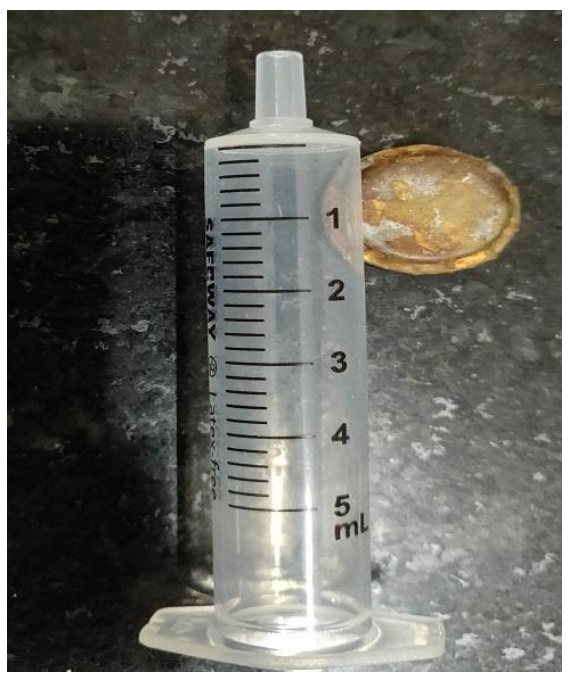

Fig.4- A metallic round body $(1.5 \mathrm{~cm}$ in diameter) removed from the nasopharynx.

There were no immediate post-operative complications.

\section{Discussion}

Foreign body lodgment in upper aero-digestive tract is a common occurrence and of all the sites known nasopharynx is one of the rare ones. The plausible reason for the same is the natural barrier offered by the nasopharyngeal sphincter and the soft palate to upward migration of the foreign body following ingestion, a vast space and also to its anatomical location which is anti-gravity. ${ }^{[3]}$ Instances of a violent bout of cough, forceful emesis, unskilful attempt at removing a foreign body lodged in oral cavity, penetrating trauma and even heimlich's maneuver has been implicated for causing lodgment of the foreign body in nasopharynx..$^{[4-7]}$ An array of foreign bodies ranging from inorganic such as a nut, metallic ring, coin, wooden piece, needle to organic ones such as a leech and fish have been reported. ${ }^{[4-}$ ${ }^{6],[8-10]}$ The youngest reported case report for foreign body nasopharynx was in an 8th month old infant. ${ }^{[4]}$ Ours is a case with foreign body in the nasopharynx, which most likely lodged there following an episode of a bout of cough in an 8 month old infant. A reliable history of foreign body ingestion, followed by proper assessment of aero-digestive tract is the key to diagnosis. Usually nasopharyngeal foreign bodies are asymptomatic, classically presenting as lost foreign bodies. Long term neglected foreign bodies present as foul smelling nasal discharge, 
mouth breathing, snoring, sometimes nasal bleed and halitosis. ${ }^{[6]}$ In our case although the child was asymptomatic, an episode of URI led to exacerbation of symptoms of upper airway obstruction which further led to the child presenting with inspiratory stridor.

Considering the rarity of the site, age of presentation and symptomatology and diagnostic dilemma involved, this is an unusual case which warrants to be reported. A nasopharyngeal foreign body missed on diagnosis is potentially precarious as it may sometimes descend to cause airway obstruction leading to fatal outcomes.

In most instances as the foreign body is radioopaque, radiological investigation is the modality of choice for confirming the diagnosis. An X-ray scan involving nasopharynx, neck and chest is usually done. Stress should be given on getting a lateral view X-ray for nasopharynx for proper delineation of the foreign body. In case of high index of suspicion, with the foreign body not visible radiologically, nasopharyngoscopy can be done for direct visualization of the foreign body.

Foreign body removal should be done under general anesthesia after proper securement of the airway with oro-tracheal intubation. ${ }^{[4],[6]}$ It can be done both trans-nasally and through trans-oral route depending upon the ease and safety of the procedure. The removal should be carried out under vision, avoiding injury to the nasopharyngeal wall which might perforate and can sometimes complicate into a retropharyngeal abscess. ${ }^{[11]}$ Bleeding and injury to the nasopharyngeal wall are other more common risks associated with the procedure.

\section{Conclusions}

Nasopharyngeal foreign bodies are rarely seen and are usually asymptomatic. A high index of suspicion is needed for diagnosing them. Radiological and endoscopic examination for visualization is the investigation needed for confirming the diagnosis. Complications leading to aspiration and airway obstruction can lead to fatal outcomes. This case needs merit as it is one of the very few cases reported in such a young age group, also the symptomatology as a likely cause for inspiratory stridor has never been discussed previously.

\section{Acknowledgement: None}

\section{References}

1. JKAG Sunkum, "Nasopharyngeal Foreign body in a Young Child," Indian J Otolaryngol Head Neck Surg, vol. 63, pp. 285-286, May 2011

2. J. Ransome, Foreign bodies in the nose, 5 th ed., A. G. Kerra and J. Groves, Eds. London, UK: Scott Brown's Otolaryngology, 1987.

3. B. Swaminathan, S. Ponnusamy, R. Shanmugam., V. Umapathy and R. Mariappan, "Long standing neglected foreign body in nasopharynx," International Journal of Current Medical Sciences, vol. 7, pp. 229-230, March 2017.

4. L. Ray, P. Chatterjee, S.N. Bandyopadhyay, S. Das , R. Sinha, T.K. Nandy, "An unusual foreign body (big metallic nut) in the nasopharynx of an infant," Indian J Otolaryngol Head Neck Surg, vol. 56, pp. 309-310, Oct. 2004

5. S. Kanotra, M. Lateef, S. Ahmed," Nasopharyngeal Foreign Bodies," Asian J Ear Nose Throat, vol. 3, pp. 21-25, January 2007.

6. P.A. Thompson, Y.A. Awuku," Metallic ring in the nasopharynx of a child simulating sinusitis-A Case Report," Case Study and Case Report, vol. 3 pp. 21-25, 2013

7. Shanmugam V.U, T. Doss, R. Shanmugam, Bharath, P. Nivas," Nasopharyngeal Foreign Body Following Heimlich Manuever- A Rare Case Report," Journal of medical science and clinical research, vol. 7, pp. 6467, March 2019.

8. P.K. Majumder, A.K. Sinha, P.B. Mookherje, S.N. Ganguly, "An unusual foreign body (10 N.P.coin) in nasopharynx," Indian J Otolaryngol Head Neck Surg, vol. 52, pp. 93, Dec. 1999. 
9. C. Bilgen, B. Karci, and U. Uluoz, "A nasopharyngeal mass: leech in the nasopharynx," International Journal of Pediatric Otorhinolaryngology, vol. 64, pp. 73-76, 2002.

10. R. D. Briggs, A. M. Pou, and N. R. Friedman, "An unusual catch in the nasopharynx," American Journal of Otolaryngology, vol. 22, pp. 354-357, 2001.

11. P.A. Onakoya, A.A. Adoga, A.S. Adoga, C. Galadima, O.G. Nwaorgu," An unusual rhino-pharyngeal foreign body," West Afr J Med, vol. 24, pp. 89-91, 2005. 